\title{
INFLUENCE OF Si-DOPING ON CARRIER LOCALIZATION OF MOCVD-GROWN InGaN/GaN MULTIPLE QUANTUM WELLS
}

\author{
Yong-Hoon Cho*, T. J. Schmidt*, S. Bidnyk*, J. J. Song*, \\ S. Keller**, U. K. Mishra**, and S. P. DenBaars** \\ *Center for Laser and Photonics Research and Department of Physics \\ Oklahoma State University, Stillwater, OK 74078 \\ **Electrical and Computer Engineering and Materials Departments \\ University of California, Santa Barbara, CA 93106
}

\section{Cite this article as: MRS Internet J. Nitride Semicond. Res. 4S1, G6.44 (1999)}

\begin{abstract}
We have systematically studied the influence of Si doping on the optical characteristics of InGaN/GaN multiple quantum wells (MQWs) using photoluminescence (PL), PL excitation (PLE), and time-resolved PL spectroscopy combined with studies of optically pumped stimulated emission and structural properties from these materials. The MQWs were grown on 1.8- $\mu$ m-thick $\mathrm{GaN}$ layers on c-plane sapphire films by metalorganic chemical vapor deposition. The structures consisted of 12 MQWs with 3-nm-thick InGaN wells, 4.5-nm-thick GaN barriers, and a 0.1- $\mu$ mthick $\mathrm{Al}_{0.07} \mathrm{Ga}_{0.93} \mathrm{~N}$ capping layer. The Si doping level in the $\mathrm{GaN}$ barriers was varied from $1 \mathrm{x}$ $10^{17}$ to $3 \times 10^{19} \mathrm{~cm}^{-3}$. PL and PLE measurements show a decrease in the Stokes shift with increasing Si doping concentration. The $10 \mathrm{~K}$ radiative recombination lifetime was observed to decrease with increasing Si doping concentration $(n)$, from $\sim 30 \mathrm{~ns}$ (for $n<1 \times 10^{17} \mathrm{~cm}^{-3}$ ) to $\sim$ ns (for $n=3 \times 10^{19} \mathrm{~cm}^{-3}$ ). To elucidate whether non-radiative recombination processes affect the measured lifetime, the temperature-dependence of the measured lifetime was investigated. The reduced Stokes shift, the decrease in radiative recombination lifetime, and the increase in structural and interface quality with increasing Si doping indicate that the incorporation of Si in the $\mathrm{GaN}$ barriers results in a decrease in carrier localization at potential fluctuations in the InGaN active regions and the interfaces.
\end{abstract}

\section{INTRODUCTION}

Group III nitride semiconductors and their heterostructures have recently attracted much attention for their versatile applications as light emitting devices, solar-blind ultraviolet detectors, and high power/high temperature/high frequency devices [1-6]. Much interest has been focused on InGaN/GaN quantum wells (QWs) because of their applications as high brightness light emitting diodes and cw blue laser diodes [1,2]. Detailed knowledge of the effects of Si doping on the optical properties of these materials is crucial, especially for designing practical devices. Recently, the effects of Si doping on the optical properties of GaN epilayers [7,8], InGaN/GaN QWs [9-11], and GaN/AlGaN QWs [12,13] have been reported. However, the influence of Si doping on both optical properties and structural qualities in InGaN/GaN multiple quantum well (MQW) device structures is presently not well understood.

In this study, we present a comprehensive study of the optical properties of InGaN/GaN MQWs using photoluminescence (PL), PL excitation (PLE), and time-resolved PL (TRPL) spectroscopy, combined with studies of optically pumped stimulated emission (SE) and structural properties from these materials. In particular, the influence of Si doping in the GaN barriers on the characteristics of the MQWs is investigated. PL and PLE results show that 
increasing the Si doping concentration $(n)$ reduces the Stokes shift of the InGaN emission. TRPL experiments show the $10 \mathrm{~K}$ recombination lifetime decreases with increasing $n$, from $\sim 30 \mathrm{~ns}$ (for $n<1 \times 10^{17} \mathrm{~cm}^{-3}$ ) to $\sim 4 \mathrm{~ns}$ (for $n=3 \times 10^{19} \mathrm{~cm}^{-3}$ ). In addition, it is shown that Si doping improves the structural and interface properties of the InGaN/GaN MQW samples from atomic force microscopy (AFM) images. From the results, we demonstrate that Si doping in the GaN barriers affects the interface quality of the InGaN/GaN MQW systems, and influences the optical properties.

\section{EXPERIMENT}

The InGaN/GaN MQWs used in this study were grown on c-plane sapphire films by metalorganic chemical vapor deposition, following the deposition of a 1.8 - $\mu$ m-thick GaN buffer layer. The structures consisted of $12 \mathrm{MQWs}$ with 3-nm-thick $\operatorname{In}_{0.2} \mathrm{Ga}_{0.8} \mathrm{~N}$ wells and 4.5-nm-thick $\mathrm{GaN}$ barriers, and a 100-nm-thick $\mathrm{Al}_{0.07} \mathrm{Ga}_{0.93} \mathrm{~N}$ capping layer. Trimethylgallium (TMGa), trimethylindium (TMIn), trimethylaluminum, and ammonia were used as the precursors and disilane was used as the $n$-type dopant. The growth temperatures of the $\mathrm{GaN}$ base layer, the superlattice (SL) region, and the AlGaN capping layer were 1050,790 , and $1040^{\circ} \mathrm{C}$, respectively. The TMGa and TMIn fluxes during the SL growth were 5 and $14 \mu \mathrm{mol} / \mathrm{min}$, respectively, while the ammonia flow was held constant at $0.35 \mathrm{~mol} / \mathrm{min}$. In order to study the influence of $\mathrm{Si}$ doping in the GaN barriers, the disilane doping precursor flux was systematically varied from 0 to $4 \mathrm{nmol} / \mathrm{min}$ during $\mathrm{GaN}$ barrier growth. Accordingly, a doping concentration in the range of < $1 \times 10^{17}$ to $3 \times 10^{19} \mathrm{~cm}^{-3}$ was achieved for the different samples, which was determined by secondary ion mass spectroscopy and Hall measurements. To investigate the effect of Si doping on the GaN surface morphology, three reference 7-nm-thick GaN epilayers were also grown at $800{ }^{\circ} \mathrm{C}$, which have different disilane flux rates of $0,0.2$, and $2 \mathrm{nmol} / \mathrm{min}$ during growth. The surface morphology of the reference samples were investigated using a Digital Instruments Nanoscope III AFM operated in tapping mode and the root-mean-square surface roughness was estimated by the AFM software. Details of the growth procedure and the other structural properties are given elsewhere [10,14]. PL and PLE spectra were measured using the $325 \mathrm{~nm}$ line of a $20 \mathrm{~mW} \mathrm{cw} \mathrm{He}-\mathrm{Cd}$ laser and the quasi-monochromatic light from a xenon lamp dispersed by a $1 / 2 \mathrm{~m}$ monochromator, respectively. TRPL measurements were carried out using a picosecond pulsed laser system consisting of a cavity-dumped dye laser for sample excitation and a streak camera for detection. The output laser pulses from the dye laser had a duration of less than 5 ps and were frequency doubled into the UV spectral region by a nonlinear crystal. The overall time resolution of the system is better than $15 \mathrm{ps}$. The temperature of the sample in the experiments described above was varied from 10 to $300 \mathrm{~K}$.

\section{RESULTS AND DISCUSSIONS}

Figure 1 shows $10 \mathrm{~K}$ PL (solid lines) and PLE (dashed lines) spectra of the main InGaNrelated PL emission peak with the decay time plotted as a function of emission energy (open circles). The near-band-edge emission from the AlGaN cladding layer and the GaN barriers are also clearly seen at 3.60 and $3.48 \mathrm{eV}$, respectively. The PLE detection energy is set at the main PL peak energy. The contributions from the GaN barriers and the AlGaN capping layer are clearly distinguishable, and the band-edge PLE positions are coincident with the PL peak positions. A large Stokes shift of the PL emission from the InGaN wells with respect to the bandedge measured by PLE is clearly observed, which is attributed to crystal imperfections such as In alloy fluctuations and interface roughness. We observed that as the Si doping concentration increases, the Stokes shift decreases. The Stokes shift for the sample with $n=3 \times 10^{19} \mathrm{~cm}^{-3}$ is 


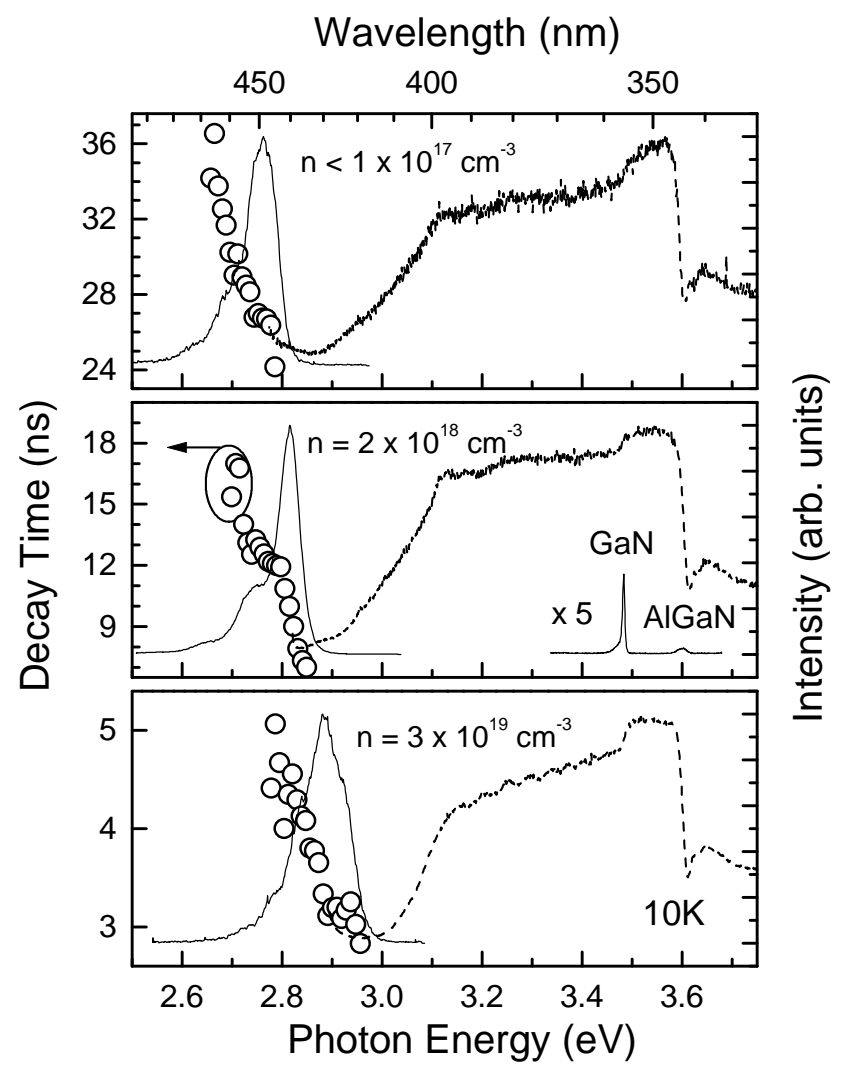

Figure 1. 10 K PL (solid lines), PLE (dashed lines), and TRPL data (open circles) of 12period InGaN/GaN MQWs with $\mathrm{Si}$ doping concentrations ranging from $<1 \times 10^{17}$ to $3 \mathrm{x}$ $10^{19} \mathrm{~cm}^{-3}$ in the GaN barriers. The Stokes shift between the PL emission peak and the absorption band-edge observed from PLE spectra decreases significantly as the Si doping concentration increases. Luminescence decay times measured by TRPL are shown as a function of emission energy. With increasing $\mathrm{Si}$ doping concentration, the $10 \mathrm{~K}$ lifetimes decrease from $~ 30 \mathrm{~ns}$ (for $n<1 \times 10^{17} \mathrm{~cm}^{-3}$ ) to $\sim 4 \mathrm{~ns}$ (for $n=3 \times 10^{19} \mathrm{~cm}^{-3}$ ).

$120 \mathrm{meV}$ smaller than that of the nominally undoped sample (see Table I). The effect of Si doping on the decay time of the MQWs was also explored using TRPL measurements. Figure 1 (open circles) shows the $10 \mathrm{~K}$ decay time $\left(\tau_{\mathrm{d}}\right)$ monitored at different emission energies. The measured lifetime becomes longer with decreasing emission energy, and hence, the peak energy of the emission shifts to the low energy side as time proceeds. This behavior is characteristic of localized states, which in this case are most likely due to alloy fluctuations and/or interface irregularities in the MQWs [15]. A decrease in $\tau_{\mathrm{d}}$ with increasing Si doping, from $~ 30 \mathrm{~ns}$ (for $n$ $<1 \times 10^{17} \mathrm{~cm}^{-3}$ ) to $\sim 4 \mathrm{~ns}$ (for $n=3 \times 10^{19} \mathrm{~cm}^{-3}$ ), is clearly seen, which can be attributed to a decrease in the potential fluctuations leading to recombination.

One should be very careful interpreting the low temperature lifetime results because the non-radiative recombination processes (which can be caused by poor sample quality) may affect the measured lifetime. To clarify this point, we investigated the temperature dependence of the lifetime as shown in Fig. 2. For the nominally undoped sample with $n<1 \times 10^{17} \mathrm{~cm}^{-3}$ (open symbols), we observed an increase of $\tau_{\mathrm{d}}$ with temperature (up to around $40 \mathrm{~ns}$ for the higher energy side emission) at temperatures below $70 \mathrm{~K}$, in qualitative agreement with the temperature dependence of radiative recombination. As the temperature is further increased beyond a certain crossover temperature, $T_{c}$, which is determined by the radiative and non-radiative recombination rates, the lifetime starts to decrease, since non-radiative processes predominantly influence the emission. Further evidence of this is given by the fact that the lifetimes become independent of emission energy at higher temperatures. We note that $T_{c}$ gradually increases as $n$ increases: $T_{c} \sim$ $70 \mathrm{~K}$ for $n<1 \times 10^{17} \mathrm{~cm}^{-3}, T_{c} \sim 100 \mathrm{~K}$ for $n=2 \times 10^{18} \mathrm{~cm}^{-3}$ (not shown here), and $T_{c} \sim 140 \mathrm{~K}$ for $n=3 \times 10^{19} \mathrm{~cm}^{-3}$. This indicates that the decrease in lifetime is due to a decrease of the radiative recombination lifetime itself rather than an increased influence of non-radiative recombination 


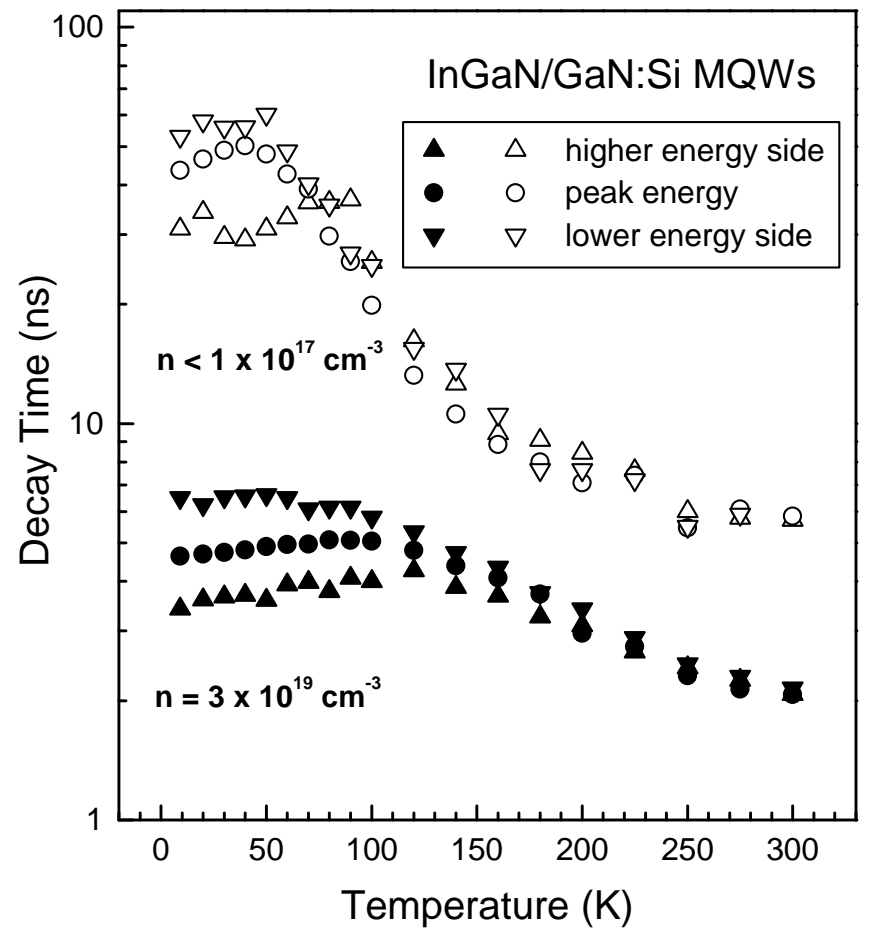

Figure 2. Temperature dependence of decay times monitored above (up triangles), below (down triangles), and at (circles) the emission peak for the InGaN/GaN MQWs with $n<1 \mathrm{x}$ $10^{17} \mathrm{~cm}^{-3}$ (open symbols) and $n=3 \times 10^{19} \mathrm{~cm}^{-3}$ (closed symbols) in the $\mathrm{GaN}$ barriers. The characteristic crossover temperature, $T_{c}$, (which is determined by the radiative and non-radiative recombination rates) gradually increases as $n$ increases: $T_{c} \sim 70 \mathrm{~K}$ for $n<1 \times 10^{17} \mathrm{~cm}^{-3}, T_{c} \sim$ $100 \mathrm{~K}$ for $n=2 \times 10^{18} \mathrm{~cm}^{-3}$ (not shown), and $T_{c}$ $\sim 140 \mathrm{~K}$ for $n=3 \times 10^{19} \mathrm{~cm}^{-3}$.

processes. Therefore, we conclude that the decrease in lifetime with increasing $n$ is mainly due to a decrease in potential localization, and hence, a decrease in the carrier migration time into the lower tail states in the MQW active regions.

To further study the influence of $\mathrm{Si}$ doping on the structural properties, AFM measurements were carried out. Figure 3 shows $2 \mu \mathrm{m} \times 2 \mu \mathrm{m}$ AFM images of three reference 7$\mathrm{nm}$-thick GaN films with different disilane flow rates during growth. The root-mean-square surface roughness estimated from AFM images was $0.49,0.42$, and $0.22 \mathrm{~nm}$ for GaN epilayers with disilane flow rates of (a) 0 , (b) 0.2 , and (c) $2 \mathrm{nmol} / \mathrm{min}$ during growth, respectively. Thus, smoother $\mathrm{GaN}$ surfaces with more homogeneous terrace length were achieved at higher $\mathrm{Si}$ doping levels. In addition, the recent high-resolution x-ray diffraction (HRXRD) study showed that as $n$ increases, the full width at half maximum (FWHM) of the higher-order SL satellite peaks narrows: the FWHM of the second order SL peaks was observed to be 589, 335, and 234 arcsec for $n<1 \times 10^{17}, n=2 \times 10^{18}$, and $n=3 \times 10^{19} \mathrm{~cm}^{-3}$, respectively (see Table I) [14].

Table I. Observed Stokes shift, measured lifetime, $T_{c}$, HRXRD FWHM, and SE threshold density for InGaN/GaN MQW samples with different $\mathrm{Si}$ doping concentration $n$ by adjusting disilane flow rate $\mathrm{F}_{\mathrm{Si}}$.

\begin{tabular}{|c|c|c|c|c|c|c|c|c|c|}
\hline \multirow{2}{*}{$n\left(\mathrm{~cm}^{-3}\right)$} & \multirow{2}{*}{$\begin{array}{c}\mathrm{F}_{\mathrm{Si}} \\
(\mathrm{nmol} \\
/ \mathrm{min})\end{array}$} & \multirow{2}{*}{$\begin{array}{l}\text { Stokes } \\
\text { shift } \\
\text { at } 10 \mathrm{~K} \\
(\mathrm{meV})\end{array}$} & \multicolumn{2}{|c|}{$\begin{array}{l}\text { Measured lifetime } \\
\text { (nsec) }\end{array}$} & \multirow{2}{*}{$\begin{array}{c}T_{c} \\
(\mathrm{~K})\end{array}$} & \multicolumn{3}{|c|}{$\begin{array}{l}\text { HRXRD FWHM } \\
\quad(\operatorname{arcsec})^{\text {a) }}\end{array}$} & \multirow{2}{*}{$\begin{array}{c}\text { SE thresholc } \\
\text { density } \\
\text { at } 300 \mathrm{~K} \\
\left.\left(\mathrm{~kW} / \mathrm{cm}^{2}\right)^{\mathrm{b}}\right)\end{array}$} \\
\hline & & & $10 \mathrm{~K}$ & $300 \mathrm{~K}$ & & SL-2 & SL-1 & SL0 & \\
\hline$<1 \times 10^{17}$ & 0 & $\sim 355$ & $24-36$ & 6 & $\sim \sim 70$ & 589 & 312 & 219 & 58 \\
\hline $2 \times 10^{18}$ & 0.26 & $\sim 305$ & $7-18$ & 5 & $\sim 100$ & 335 & 276 & 229 & 55 \\
\hline $3 \times 10^{19}$ & 4 & $\sim 235$ & $3-5$ & 2 & $\sim 140$ & 234 & 212 & 227 & 165 \\
\hline
\end{tabular}

a) see Ref. [14]

b) see Ref. [11] 


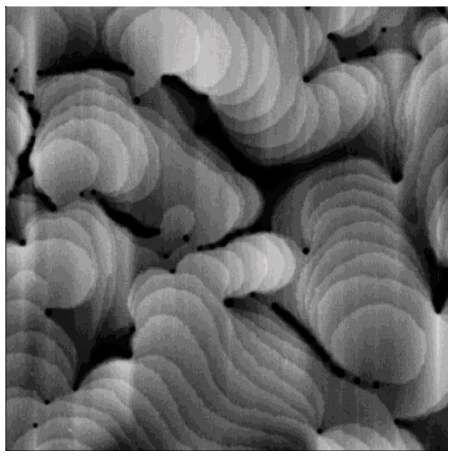

(a)

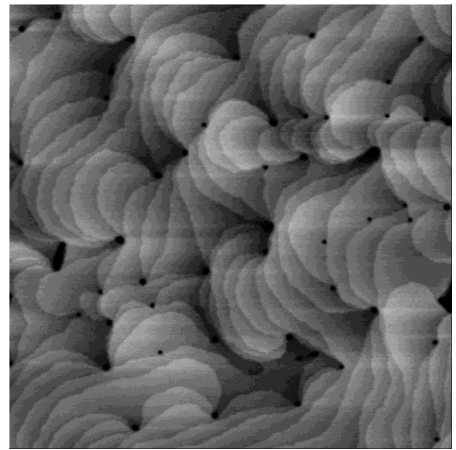

(b)

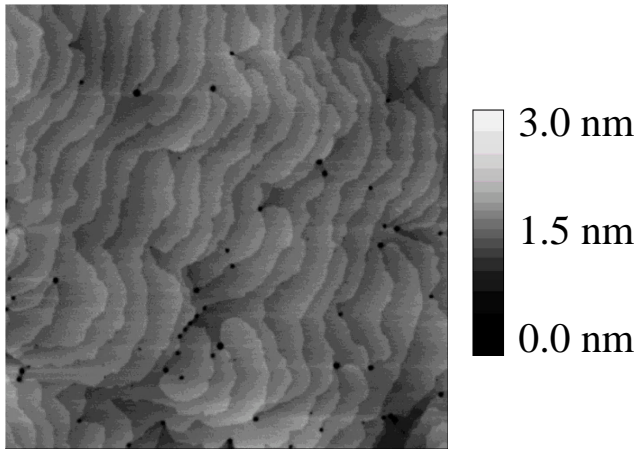

(c)

Figure 3. $2 \mu \mathrm{m} \times 2 \mu \mathrm{m}$ AFM images of three 7-nm-thick GaN films with different disilane flow rates during growth. The root-mean-square surface roughness estimated from AFM images was 0.49, 0.42, and $0.22 \mathrm{~nm}$ for $\mathrm{GaN}$ epilayers with the disilane flow rate of (a) 0 , (b) 0.2 , and (c) $2 \mathrm{nmol} / \mathrm{min}$, respectively. A higher quality $\mathrm{GaN}$ surface morphology and a larger average terrace length were achieved by increasing Si incorporation.

Accordingly, we conclude that $\mathrm{Si}$ doping in the GaN barriers significantly improves the structural and interface quality of the InGaN/GaN MQWs. Since the incorporation of Si atoms can change the quality and/or surface free energy of the GaN barriers, it may affect the growth condition (or mode) of the subsequent InGaN wells and the interfaces $[7,16,17]$. The observed experimental results such as (i) a decrease in Stokes shift and (ii) a decrease in radiative recombination lifetime with increasing $\mathrm{Si}$ doping can be explained in terms of a decrease in potential fluctuations and enhancement of the interfacial structural qualities with incorporation of $\mathrm{Si}$ in the GaN layers.

Finally, we note that the influence of Si doping on the emission efficiency and the SE threshold pump density was also investigated for the InGaN/GaN MQWs with different Si doping concentration in the GaN barriers [11]. For all the samples, power dependent PL studies show a strong blueshift of the spontaneous emission with increasing excitation density mainly due to band filling of the energy tail states. The blueshift is observed to cease shortly before the onset of SE [18]. The SE spectrum for the MQW sample with $n=2 \times 10^{18} \mathrm{~cm}^{-3}$ was composed of many narrow peaks of less than $1 \AA$ FWHM and there was no noticeable broadening of the SE peaks when the temperature was tuned from 175 to $575 \mathrm{~K}$. Interestingly, only moderately $\mathrm{Si}$ doped MQW samples exhibited enhanced luminescence efficiency and a reduction of the SE threshold (see Table I). That is, the maximum emission intensity and the lowest SE threshold (55 $\mathrm{kW} / \mathrm{cm}^{2}$ ) were achieved for the sample with $n=2 \times 10^{18} \mathrm{~cm}^{-3}$, while a SE threshold of 165 $\mathrm{kW} / \mathrm{cm}^{2}$ was obtained for the sample with $n=3 \times 10^{19} \mathrm{~cm}^{-3}$. This fact, combined with the results presented in this paper, indicates that although Si doping improves the interface properties, it does not necessarily enhance the SE properties, reflecting the fact that localized carriers at potential fluctuations may play an important role in the emission from the InGaN/GaN MQW structures used for current state-of-art laser diodes.

\section{CONCLUSIONS}

We have investigated the optical properties of InGaN/GaN MQW structures as a function of Si doping concentration in the GaN barriers. As the Si doping of the GaN barriers increases, the Stokes shift of the PL emission peak with respect to the PLE band-edge was found to decrease. In addition, the radiative recombination lifetime measured at $10 \mathrm{~K}$ was observed to decrease significantly from $\sim 30 \mathrm{~ns}$ for $n<1 \times 10^{17} \mathrm{~cm}^{-3}$ to $\sim 4 \mathrm{~ns}$ for $n=3 \times 10^{19} \mathrm{~cm}^{-3}$ with increasing $n$. The results presented here, combined with the observed structural properties, are well explained 
by a decrease in carrier localization at potential fluctuations with increasing Si doping of the $\mathrm{GaN}$ barriers. These potential fluctuations are most likely a result of In alloy fluctuations and/or thickness variations of the InGaN well regions. The influence of $\mathrm{Si}$ doping on the emission efficiency and stimulated emission threshold was also discussed.

\section{ACKNOWLEDGMENTS}

This work was supported by AFOSR, ARO, ONR, DARPA, and KOSEF.

\section{REFERENCES}

1. S. Nakamura, M. Senoh, N. Iwasa, S. Nagahama, T. Yamada, and T. Mukai, Jpn. J. Appl. Phys. Part 2 34, L1332 (1995).

2. S. Nakamura, M. Senoh, S. Nagahama, N. Iwasa, T. Yamada, T. Matsushita, Y. Sugimoto, and H. Kiyoku, Appl. Phys. Lett. 69, 4056 (1996).

3. B. W. Lim, Q. C. Chen, J. Y. Yang, and M. A. Khan, Appl. Phys. Lett. 68, 3761 (1996).

4. Y. F. Wu, B. P. Keller, S. Keller, D. Kapolnek, P. Kozodoy, S. P. DenBaars, and U. K. Mishra, Appl. Phys. Lett. 69, 1438 (1996).

5. X. H. Yang, T. J. Schmidt, W. Shan, J. J. Song, and B. Goldenberg, Appl. Phys. Lett. 66, 1 (1995).

6. H. Morkoç, S. Strite, G. B. Gao, M. E. Lin, B. Sverdlov, and M. Burns, J. Appl. Phys. 76, 1363 (1994).

7. S. Ruvimov, Z. Liliental-Weber, T. Suski, J. W. Ager III, J. Washburn, J. Krueger, C. Kisielowski, E. R. Weber, H. Amano, and I. Akasaki, Appl. Phys. Lett. 69, 990 (1996).

8. E. F. Schubert, I. D. Goepfert, W. Grieshaber, and J. M. Redwing, Appl. Phys. Lett. 71, 921 (1997).

9. P. A. Grudowski, C. J. Eiting, J. Park, B. S. Shelton, D. J. H. Lambert, and R. D. Dupuis, Appl. Phys. Lett. 71, 1537 (1997).

10. S. Keller, A. C. Abare, M. S. Minsky, X. H. Wu, M. P. Mack, J. S. Speck, E. Hu, L. A. Coldren, U. K. Mishra, and S. P. DenBaars, Materials Science Forum 264 - 268, 1157 (1998).

11. S. Bidnyk, T. J. Schmidt, Y. H. Cho, G. H. Gainer, J. J. Song, S. Keller, U. K. Mishra, and S. P. DenBaars, Appl. Phys. Lett. 72, 1623 (1998).

12. A. Salvador, G. Liu, W. Kim, O. Aktas, A. Botchkarev, and H. Morkoç, Appl. Phys. Lett. 67, 3322 (1995).

13. K. C. Zeng, J. Y. Lin, H. X. Jiang, A. Salvador, G. Popovici, H. Tang, W. Kim, and H. Morkoç, Appl. Phys. Lett. 71, 1368 (1997).

14. Y. H. Cho, F. Fedler, R. J. Hauenstein, G. H. Park, J. J. Song, S. Keller, U. K. Mishra, and S. P. DenBaars, J. Appl. Phys. (to be published).

15. Y. H. Cho, G. H. Gainer, A. J. Fischer, J. J. Song, S. Keller, U. K. Mishra, and S. P. DenBaars, Appl. Phys. Lett. 73, 1370 (1998).

16. H. J. Osten, J. Klatt, G. Lippert, B. Dietrich, and E. Bugiel, Phys. Rev. Lett. 69, 450 (1992).

17. D. J. Eaglesham, F. C. Unterwald, and D. C. Jacobson, Phys. Rev. Lett. 70, 966 (1993).

18. T. J. Schmidt, Y. H. Cho, G. H. Gainer, J. J. Song, S. Keller, U. K. Mishra, and S. P. DenBaars, Appl. Phys. Lett. 73, 560 (1998); ibid. 73, 1892 (1998). 